\title{
Need for training in clinical research for postgraduate trainees
}

\author{
Kamani Wanigasuriya ${ }^{1}$ \\ Journal of the Ceylon College of Physicians, 2011, 42, 16-20
}

\begin{abstract}
Objectives: Postgraduate training programme leading to MD (Medicine) encourages trainees to do research during their training period and a research project is a mandatory component for senior registrar training period in General Medicine. Postgraduate trainees' perceptions towards clinical research and factors associated with their enthusiasm to participate in research were assessed in the present study.
\end{abstract}

Method: A self administered questionnaire was sent to 110 trainees between March 2011 and June 2011 to collect descriptive data on resources available for research, perceptions and research productivity.

Results: Response rate was $72 \%$. Of the senior registrars, $71 \%$ were of the view that the research should be a mandatory component in training programme. Non availability of a research curriculum and lack of financial support were the two main institutional barriers to undertaking research identified by the trainees. Lack of time due to heavy clinical commitments and inadequacy of research training were identified as the two main personal obstacles. The opportunities for trainees to receive formal training and the research productivity were found to be inadequate.

Conclusions: In order to achieve maximum benefits from the research component, it should be supported with a formal training in research, research curriculum, protected time and good mentorship.

\section{Introduction}

Research has become an integral part of modern medical practice. The physician should keep abreast of latest developments in medicine as well as contribute to the new medical knowledge to provide a

\footnotetext{
${ }^{1}$ Senior Lecturer, Department of Medicine, Faculty of Medical Sciences, University of Sri Jayewardenepura, Sri Lanka.
}

better and comprehensive care to the patients. Due to the scarcity of available local literature, most developing countries use guidelines developed in western countries to manage many diseases. Hence contribution of new knowledge to medical literature is even more important in developing countries to provide cost effective patient care. The obstacles to biomedical and health care research in developing countries are complex and often poorly understood. The overall research output stands very low in Sri Lanka compared to other Asian countries ${ }^{1,2}$. Fostering a research culture among the postgraduate trainees will enhance the future research output in the country.

Postgraduate training in General Medicine consists of two and half years of training at registrar level and two to three year training depending on the specialty at senior registrar level after the MD examination. In order to encourage clinical research among postgraduate trainees, the postgraduate training programme in General Medicine has recently introduced research as a compulsory component of senior registrar training.

The objective of the present study was to investigate both the perception of postgraduate trainees in Medicine and related specialties towards clinical research and factors associated with their enthusiasm to participate in research. In addition, we aimed to identify their future perspective in relation to research. Findings of this study would help to improve the training to promote a research culture and to identify other factors that promote research in young physicians.

\section{Methods}

There were 151 trainees in the MD (Medicine) programme at the time of survey. One hundred and ten trainees who have completed six months of training in five teaching hospitals were recruited. The study was carried out during the period of March to June 2011. A 45 item, validated questionnaire modified to suit the local needs were posted to them ${ }^{3}$. This questionnaire has been designed to collect descriptive data on perceptions, productivity and resources available for research. Ethical approval was obtained from the Ethical Committee of the Faculty of Medical Sciences and permission to carry out the research 
was obtained from the Director, Postgraduate Institute of Sri Lanka.

\section{Results}

A total of 82 questionnaires were returned with a response rate of $74 \%$. Table 1 presents the demographic data of trainees stratified by the level of training; senior registrar (post MD) and registrar (pre$M D$ ). Out of the 82 responded 35 were senior registrars (21 in General Medicine and 14 in other specialties) and 47 were registrars. The majority of responders were working in the units under the Ministry of Health while the rest were working in University units.

Forty two percent of trainees (51.4\% senior registrars and $36.2 \%$ registrars) were currently involved in a research project. Of the senior registrars, 25 out of $35(71 \%)$ were of the view that the research should be a mandatory component in training programme compared to 26 out of 47 registrars (55\%) who had the same view. The majority of trainees (87\%) agreed that involvement in research helps to understand scientific principles and methods while almost $96 \%$ agreed that as a member of the medical community they should add to the existing medical knowledge. Only $56(68.3 \%)$ trainees were interested in taking up an academic career in the future while a higher proportion 67 (81\%) of trainees expressed that they are likely to undertake clinical research while they practice as consultant physicians. Of all respondents, $61(74 \%)$ agreed that it is important to learn how to apply research findings in journals to clinical practice.

Forty four percent of trainees (47\% registrars and $40 \%$ senior registrars) had some research experience prior to enrolment to the training programme and one registrar has obtained a postgraduate degree in research. Only 9 trainees (10.8\%) admitted that the research experience they had during their undergraduate training as adequate.

When asked to rank three or more institutional and personal barriers to undertaking research during the training period, non availability of a research curriculum and lack of financial support were the two main institutional barriers identified. Lack of time due to heavy clinical commitments and inadequacy of research training were identified as personal obstacles by most of the responders (Table 3). Most trainees $(80 \%)$ felt that there was inadequate time available for research work. Ninety two percent felt that it is important to have a protected time allocated for research during the training period. Regarding the basic skills training, $52(63.8 \%)$ of trainees had not attended a research methodology course and $67(81.7 \%)$ of trainees has not had the opportunity to attend a basic IT course at the time of survey.

Of all trainee respondents, 24 (29\%) had at least one published research material. During the training period $13(21.7 \%)$ had presented a poster in an academic conference while 11 (19\%) had done oral presentations (Table 2). Out of the 24 trainees who had published research 19 (79.2\%) were married and $20(83.3 \%)$ were responsible for caring for children or dependants.

Research support available in the unit is shown in Figure 1. Trainee perceptions on supervisors and research guidance available in the units are given in Figure 2.

Table 1. Demographic characteristics of postgraduate trainees

\begin{tabular}{|c|c|c|c|c|}
\hline & & $\begin{array}{c}\text { Total } \\
\text { No. (\%) }\end{array}$ & $\begin{array}{c}\text { Senior Registrar } \\
\text { No. (\%) }\end{array}$ & $\begin{array}{l}\text { Registrar } \\
\text { No. (\%) }\end{array}$ \\
\hline \multirow[t]{3}{*}{ 1) } & Place of work & & & \\
\hline & a) Health Ministry Units & $63(76.8 \%)$ & $25(71.4 \%)$ & $38(80.9 \%)$ \\
\hline & b) University Units & $19(23.2 \%)$ & $10(28.6 \%)$ & $9(19.1 \%)$ \\
\hline \multirow[t]{3}{*}{ 2) } & Marital status & & & \\
\hline & a) Married & $65(78.3 \%)$ & $30(85.70 \%)$ & $35(72.90 \%)$ \\
\hline & b) Single & $18(21.7 \%)$ & $5(14.30 \%)$ & $13(27.10 \%)$ \\
\hline \multirow[t]{3}{*}{ 3) } & Dependents & & & \\
\hline & a) Present & 69 (83.10\%) & $29(82.90 \%)$ & $40(83.30 \%)$ \\
\hline & b) None & $13(16.90 \%)$ & $6(17.10 \%)$ & $7(16.70 \%)$ \\
\hline \multirow[t]{3}{*}{ 4) } & University entry & & & \\
\hline & a) Colombo district & $16(20 \%)$ & $8(22.90 \%)$ & $8(17.80 \%)$ \\
\hline & b) Other districts & $64(80 \%)$ & $27(77.10 \%)$ & $37(82.20 \%)$ \\
\hline
\end{tabular}


Table 2. Publications/ abstracts by the trainees

\begin{tabular}{lcccc}
\hline & \multicolumn{2}{c}{ Senior Registrar } & \multicolumn{2}{c}{ Registrar } \\
\cline { 2 - 5 } & Yes & No & Yes & No \\
\hline Publication as a paper & 11 & 9 & 13 & 17 \\
& $(55 \%)$ & $(45 \%)$ & $(43.3 \%)$ & $(56.7 \%)$ \\
Poster presentation during the training period & 13 & 17 & 10 & 20 \\
& $(43.3 \%)$ & $(56.7 \%)$ & $(33.3 \%)$ & $(66.7 \%)$ \\
Abstract submitted during the training period & 13 & 17 & 8 & 20 \\
& $(43.3 \%)$ & $(56.7 \%)$ & $(28.6 \% 0$ & $(71.4 \%)$ \\
\hline
\end{tabular}

Table 3. Barriers for undertaking research during PG training

\begin{tabular}{llll}
\hline & $\begin{array}{c}\text { Registrar } \\
(\mathrm{n} \text { 35) }\end{array}$ & $\begin{array}{c}\text { Senior registrar } \\
(\mathrm{n} \text { 47) }\end{array}$ & Total \\
\hline $\begin{array}{l}\text { 1. Institutional reasons } \\
\text { Lack of a research training }\end{array}$ & $19(40.4 \%)$ & $07(20 \%)$ & $26(31.7 \%)$ \\
Non availability of financial support & $09(19.1 \%)$ & $11(31.4 \%)$ & $20(24.4 \%)$ \\
Inadequate resources in the unit & $05(10.6 \%)$ & $08(22.9 \%)$ & $13(15.9 \%)$ \\
Difficulty in continuing during short appointments & $04(8.5 \%)$ & $01(2.9 \%)$ & $05(6.1 \%)$ \\
\hline 2. Personal reasons & & & \\
Clinical commitments takes up all time & $26(55.3 \%)$ & $12(34.3 \%)$ & $38(46.3 \%)$ \\
Inadequate research training & $06(12.8 \%)$ & $06(17.1 \%)$ & $12(14.6 \%)$ \\
Personal commitment to family & $04(8.5 \%)$ & $07(20 \%)$ & $11(13.4 \%)$ \\
Time constraints due to other personal reasons & $06(12.8 \%)$ & $05(14.3 \%)$ & $11(13.4 \%)$ \\
\hline
\end{tabular}

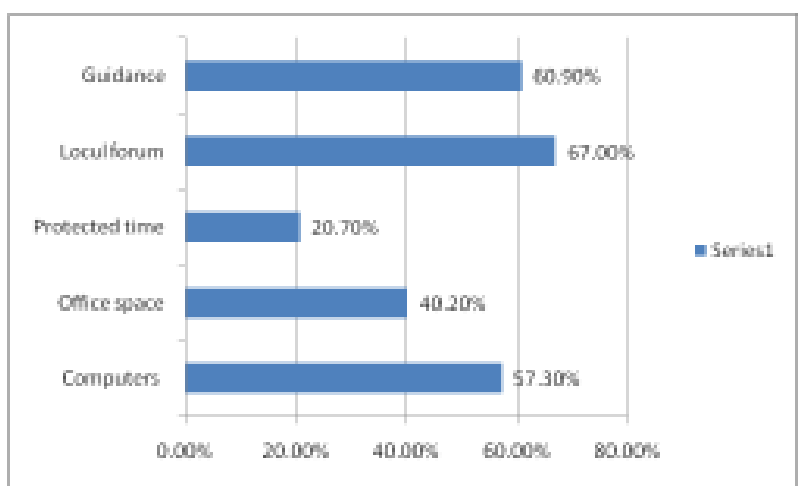

Figure 1. Support for research available in the unit.

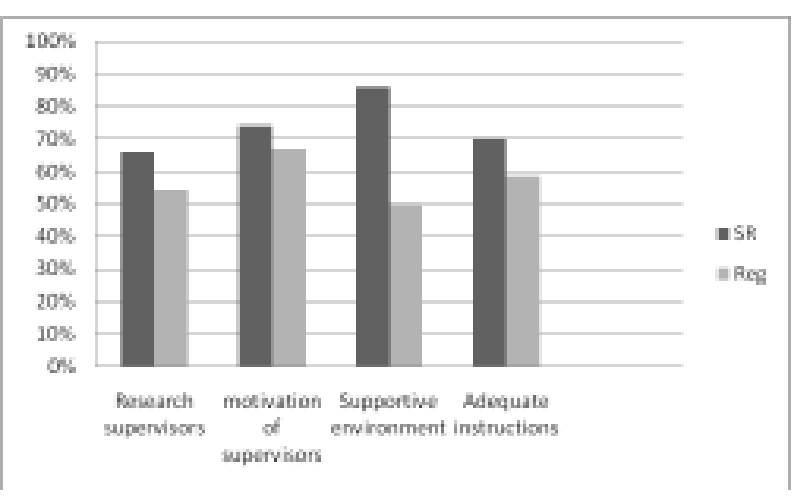

Figure 2. Perceptions of trainees regarding their supervisors. 


\section{Discussion}

Clinical research in Sri Lanka is developing at a slow pace mainly due to the lack of proper training and clinical investigators, especially of physicianscientists. There is a need for improving the manpower capacity for clinical research by improving the knowledge base of scientific research among the future physicians. Hence, the institutions devoted for medical education such as medical faculties and the Postgraduate Institute have the responsibility of training medical graduates and young physicians in research work. Postgraduate training programme leading to MD (Medicine) encourages trainees to do research during their training period and research project is a mandatory component for senior registrars in the post MD training period in General Medicine. The objective is to provide an opportunity to learn the fundamental skills needed for clinical research. This would help to improve the quality of research output by the future physicians and also improve the critical appreciation of published research.

While majority of senior registrars agreed that a research project should be a mandatory component in training, 54\% registrars supported this change. Findings of this study show that the most trainees were aware of the importance of research. Majority (87\%) agreed that involvement in research helps to understand scientific principles and methods and almost all agreed that as a member of medical community they should add to the existing medical knowledge. Further, publications or studies in progress will be beneficial for senior trainees in applying for overseas jobs. Similar observations were reported by Hayward et al in a survey of 112 alumni and 92 current residents at a residency programme that requires original research. Most alumni felt that the research project was a valuable learning experience, particularly in improving their abilities to critically review the medical literature. Almost a third felt that it had influenced their career choices ${ }^{4}$.

Research capabilities are duly recognized by academic promotion and selection committees in universities, but no priority is given for research in the promotion scheme of physicians in the Ministry of Health $(\mathrm{MOH})$. Yet majority of trainees who would be future consultants in the $\mathrm{MOH}$ expressed that they are likely to undertake clinical research in the future indicating their interest in research.

Segal et al found medical school research experience to be strongly associated with postgraduate research involvement ${ }^{5}$. To alleviate the lack of interest in research methodology and writing, Croatia introduced a mandatory course in scientific metho- dology and communication in to the medical curriculum of their largest medical school and few years later they observed a positive attitude of students toward scientific research and significant number of students carrying out research projects and publishing scientific papers $^{6}$. Less than half in our study had limited experience in research before they entered to the MD programme. Research project is a curriculum requirement in most medical faculties in Sri Lanka and it provides an opportunity to gain research experience at an early stage of undergraduate career. Most trainees felt that research training they received as undergraduates was inadequate indicating the need for further training.

Characteristics of successful research programmes as shown by several studies include programme director, support of research, time for research, faculty involvement, a research curriculum, professional support and opportunities for presenting research $^{7-9}$. Daily workload of postgraduate trainees restrains their engagement in research activities. In the present study, lack of time was the most common obstacle to research as perceived by the trainees. This is consistent with other studies which examined similar aspects in developed countries ${ }^{3,9,10}$. Interestingly our trainees did not perceive their family commitments as a barrier to research and majority of those who had published their research had responsibilities for their families and dependants.

Non availability of a research curriculum and lack of financial support were the two main institutional barriers for research identified by the trainees. Formal training is now almost a necessity to acquire the methodological and analytical skills necessary to carry out present day clinical research which has become more complex and specialized over the years. Therefore, trainees should have the opportunity to receive formal training in the design of clinical research projects, hypothesis development, biostatistics, epidemiology, and the legal and ethical issues related to clinical research. Our survey has clearly shown that the opportunities for trainees to receive formal training were inadequate. Scarcity of research funding in Sri Lanka is another barrier for carrying out good quality research by the trainee physicians.

Although the trainees perceived that the adequate supervision and guidance is available from the mentors, there is a need for adequately prepare the faculty for research mentoring. The faculty members are invaluable in providing assistance but their clinical and academic commitment may constrain the time for such support. Collaboration with academic university departments may be useful to address this issue. Mills et al identified the availability of a research mentor along with more 
faculty research activities are contributing to the research productivity of family practice residents ${ }^{11}$.

Publications are considered as a measure of good quality and outcome of research. Only one fourth of respondents had the opportunity of publishing at least one article up to the time of survey. In general, most trainees start publishing non-research articles such as case reports as the first step but those who work in research oriented units may get the opportunity to contribute to original research papers. Scientific writing workshops conducted by the editors of local journals and experienced researches targeting those who have completed some research have shown to be helpful in improving the number and quality of publications by the trainees ${ }^{6}$.

It is often argued that carrying out even a small research study is helpful as it would help the doctors to gain experience in critical appraisal of published research. When trainees ill equipped with skills are compelled to carry out research, the outcome may be of low quality and publications of poorly designed studies could lead to undesirable consequences ${ }^{12}$. Research allowance recently proposed by the government could lead to similar consequences unless a proper method to scrutinize such proposals and progress is in place. Considering the present constraints in engaging in clinical research, it is equally important to give them training in critical appraisal of research in order to recognize good research publications and be able to apply research in to practice. Journal clubs have been a platform over the years to provide an opportunity to learn these fundamental skills. Evidence suggests that journal clubs improve reading habits, knowledge in epidemiology and statistics, skills in critical appraisal and improved ability to use evidence into practice ${ }^{13,14}$. Recommendations and guidance are emerging how to improve the conduct of journal clubs. Workshops and didactic lectures are other methods of refining critical appraisal skills of trainees.

\section{Conclusions}

Several constraints for physician trainees in engaging in clinical research have been identified in the present study. Non availability of a research curriculum, lack of financial support, lack of time and inadequacy of research training were the main obstacles identified by the trainees. In order to achieve the maximum benefit from the research component, formal training course of didactic lectures and workshops should be incorporated into the MD training programme. Research component should also be supported with a research curriculum, protected time and good mentorship. Unless an interest and motivation in clinical research is created among the trainee physicians, just making a research project compulsory may fail to achieve the objectives at the end.

\section{References}

1. Data and Statistics, National Research Council of Sri Lanka. URL; http://www.nrc.gov.lk/index.php?option=com_ content\&view=article\&id=7\&ltemid=586. Last accessed on 02.01.2012.

2. Sri Lanka Science, Technology and Innovation Statistical Handbook, NSF 2008.

3. Silcox LC, Ashbury TL, VanDenKerkhof EG, Milne B. Residents' and Program Directors' attitudes towards research during Anesthesiology training: a Canadian perspective. Anesthesia and Analgesia 2006; 102: 859-64

4. Hayward RA, Taweel F. Data and the internal medicine house officer: alumni's views of the educational value of a residency program's research requirement. Journal of General Internal Medicine 1993; 8: 140-2.

5. Segal S, Lloyd T, Houts PS, Stillman PL, Jungas RL, Greer RB. The association between students' research involvement in medical school and their postgraduate medical activities. Academic Medicine 1990; 65: 530-3.

6. MarušiC A, MarušiC M. Teaching students how to read and write science: a mandatory course on scientific research and communication in medicine. Academic Medicine 2003; 78: $1235-9$.

7. Temte JL, Hunter PH, Beasley JW. Factors associated with research interest and activity during family practice residency. Family Medicine 1994; 26: 93-7.

8. Leahy N, Sheps J, Tracy CS, Nie JX, Moineddin R, Upshur REG. Family physicians' attitudes toward education in research skills during residency. Findings from a national mailed survey. Canadian Family Physician 2008; 54: 3413-4

9. DeHaven MJ, Wilson GR, O'Connor-Kettlestrings P. Creating a research culture: what we can learn from residencies that are successful in research. Family Medicine 1998; 30: 501-7.

10. Seehusen DA, Weaver SP. Resident research in family medicine: where are we now?. Family Medicine 2009; 41: 663-8.

11. Miils OF, Zyzanski SJ, Flocke SF. Factors associated with research productivity in family practice residences. Family Medicine 1997; 27: 188-93

12. Altman DG. The scandal of poor medical research. We need less research, better research, and research done for the right reasons. British Medical Journal 1994; 308: 283-4.

13. Ebbert JO, Montori VM, Schultz HJ. The Journal Club in postgraduate medical education: a systematic review. Medical Teacher 2001; 23: 455-61

14. Harris J, Kearley K, Heneghan C, Meats E, Roberts N, Perera $\mathrm{R}$, Kearley-Shiers K. Are journal clubs effective in supporting evidence based decision making? A systematic review. Medical Teacher 2011; 33: 9-23. 